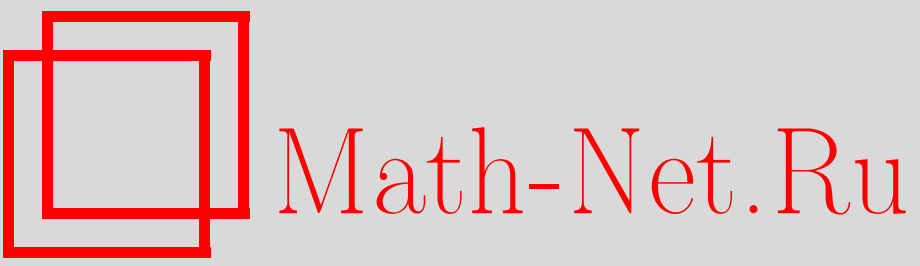

М. Н. Саушкин, А. Ю. Куров, Конечно-элементное моделирование распределения остаточных напряжений в сплошных упроченных цилиндрических образцах и образцах с полукруглым надрезом, Вестн. Сам. гос. техн. ун-та. Сер. Физ.мат. науки, 2011, выпуск 3(), 72-78

DOI: https://doi.org/10.14498/vsgtu963

Использование Общероссийского математического портала Math-Net.Ru подразумевает, что вы прочитали и согласны с пользовательским соглашением http: //www . mathnet.ru/rus/agreement

Параметры загрузки:

IP : 3.93 .64 .190

26 апреля 2023 г., 14:12:58 
УДК 539.319

\title{
КОНЕЧНО-ЭЛЕМЕНТНОЕ МОДЕЛИРОВАНИЕ РАСПРЕДЕЛЕНИЯ ОСТАТОЧНЫХ НАПРЯЖЕНИЙ В СПЛОШНЫХ УПРОЧЕННЫХ ЦИЛИНДРИЧЕСКИХ ОБРАЗЦАХ И ОБРАЗЦАХ С ПОЛУКРУГЛЫМ НАДРЕЗОМ
}

\section{М. Н. Саушкин, А.Ю. Куров}

Самарский государственный технический университет, 443100, Самара, ул. Молодогвардейская, 244.

E-mails: msaushkin@gmail.com, alexeykurov@gmail.com

\begin{abstract}
Приводится метод решения задачи о распределении остаточных напряжений в сплошных поверхностно упроченных иилиндрических образцах и образцах с полукруглым надрезом, основанный на методе конечных элементов. В качестве исходной информации используется одна и/или две экспериментально определённые компоненты остаточных напряжений в упрочнённом слое. На примере образиов из стали 45 и сплава Д16Т проведено сравнение результатов расчётов, выполненных по предложенному методу, с эксперименталъными данными и результатами расчётов по другим методикам.
\end{abstract}

Ключевые слова: распределение остаточных напряжений, иилиндрический образеи, образеи, с полукруглым надрезом, метод конечных элементов.

Введение. В последнее время для решения краевых задач механики поверхностно упрочнённых элементов конструкций широко используется метод конечных элементов (МКЭ). Определённые успехи в этом направлении достигнуты для процедуры термопластического упрочнения [1]. Имеются единичные попытки решения краевых задач для методов поверхностного пластического деформирования (обкатка роликом, алмазного выглаживания, обработка микрошариками, гидродробеструйная обработка и т. д.) на основе решения упругопластических контактных краевых задач [2-5]. Однако такие решения справедливы лишь для однократного взаимодействия обрабатываемого элемента конструкции и деформирующего элемента, что совершенно не наблюдается в реальных технологиях, где контакт в определённой точке является многократным и случайным процессом.

Поэтому для определения остаточных напряжений часто используют моделирование при заданных начальных остаточных пластических деформациях. Встречающиеся в научной литературе методы решения этой задачи для цилиндрических образцов не лишены недостатков, которые связаны с вводимыми упрощающими предположениями. Во-первых, поскольку остаточные пластические деформации после процедуры упрочнения неизвестны, то считается, что они имеют изотропное распределение по глубине слоя, что противоречит реальной картине. Во-вторых, во многих работах вместо осесимметричной задачи рассматривается плоская задача для осевого сечения цилиндрической детали.

Саушкин Михаил Николаевич (к.ф.-м.н., доц.), докторант, каф. прикладной математики и информатики. Алексей Юръевич Куров, студент, каф. прикладной математики и информатики. 
Целью настоящей работы является разработка лишённого этих недостатков метода решения поставленной задачи для упрочнённого гладкого цилиндрического образца и образца с полукруглым надрезом, нанесённым после опережающего пластического деформирования (ОПД), с учётом реального распределения полей остаточных пластических деформаций.

Под ОПД здесь понимается следующая процедура: после упрочнения в образце возникает радиально неоднородное поле остаточных пластических деформаций и неоднородное поле остаточных напряжений, а после нанесения надреза (фактически - удаления части объёма) под действием остаточных пластических деформаций, играющих роль начальных деформаций, в упрочнённом поверхностном слое происходит перераспределение остаточных напряжений.

1. Решение задачи для цилиндрического образца. Решение краевой задачи МКЭ для цилиндрического образца складывается из нескольких этапов.

Первый этап - геометрическое разбиение цилиндра на конечные элементы. На этом этапе необходимо учитывать тот факт, что остаточные напряжения обладают существенным градиентом в приповерхностном слое, составляющем 100-400 мкм (в зависимости от технологии упрочнения), что приводит к необходимости создания достаточно мелкой конечноэлементной сетки.

На втором этапе для гладкого образца моделируются начальные остаточные пластические деформации на основании аналитического решения в цилиндрической системе координат [6]:

$$
\begin{gathered}
\sigma_{r}(r)=\frac{1}{r} \int_{0}^{r} \sigma_{\theta}(\xi) d \xi \\
q_{\theta}(r)=\frac{(1+\mu)(1-2 \mu)}{E(1+\alpha \mu)^{2}} r^{-\frac{2+\alpha}{1+\alpha \mu}} \int_{0}^{r} \xi^{\frac{1+\alpha-\alpha \mu}{1+\alpha \mu}}\left[\sigma_{r}(\xi)+(1+\alpha) \sigma_{\theta}(\xi)\right] d \xi- \\
-\frac{1+\mu}{E(1+\alpha \mu)}\left[(1-\mu) \sigma_{\theta}(r)-\mu \sigma_{r}(r)\right], \\
q_{z}(r)=\alpha q_{\theta}(r), \quad q_{r}(r)=-(1+\alpha) q_{\theta}(r), \\
\varepsilon_{z}^{0}=\frac{2}{R^{2}} \int_{0}^{R} \xi\left\{q_{z}(\xi)-\frac{\mu}{E}\left[\sigma_{r}(\xi)+\sigma_{\theta}(\xi)\right]\right\} d \xi, \\
\sigma_{z}(r)=E\left(\varepsilon_{z}^{0}-q(r)\right)+\mu\left(\sigma_{r}(r)+\sigma_{\theta}(r)\right),
\end{gathered}
$$

где $\mu, E$ - упругие константы материала; $R$ - радиус цилиндра; $\sigma_{r}(r), \sigma_{\theta}(r)$, $\sigma_{z}(r)$ - радиальное, окружное и осевое остаточные напряжения; $q_{r}(r), q_{\theta}(r)$, $q_{z}(r)$ - соответствующие остаточные пластические деформации; $\alpha$ - параметр анизотропии упрочнения $(0<\alpha<\infty)$.

Отметим, что аналитическое решение (1)-(5) строится в предположении, что a priori известно распределение окружной компоненты $\sigma_{\theta}(r)$ по радиусу во всём образце и известен параметр $\alpha$. При этом считается, что в образце после упрочнения не возникали вторичные пластические деформации в области сжатия. Разработана методика [6], которая позволяет построить такое решение по одной и/или двум компонентам остаточных напряжений в упрочнённом слое, определённым экспериментально в области сжатия.

Другими словами, на втором этапе в цилиндрическом образце задаются начальные остаточные пластические деформаций $q_{i}(r)$, которые определя- 
ются аналитически по формулам (2), (3), на основании экспериментальной информации о распределении одной и/или двух компонент остаточных напряжений в упрочнённом слое. В настоящей работе они моделируются псевдотемпературными деформациями:

$$
q_{i}(r)=\alpha_{i}(T)\left(T(r)-T_{0}\right), \quad i=r, \theta, z, \quad 0 \leqslant r \leqslant R,
$$

где $T_{0}=$ const - начальная температура, а закон изменения $T=T(r)$ задаётся линейной (или любой другой) функцией. Тогда, зная $q_{i}(r)$, из $(6)$ определяется функция $\alpha_{i}=\alpha_{i}(T(r))$ как функция радиуса.

Далее, на третьем этапе, решается задача фиктивной термоупругости относительно неизвестных остаточных напряжений.

На основании предложенного подхода разработан совместимый с вычислительным комплексом ANSYS программный продукт, автоматизирующий решение поставленной задачи. Сначала на основании экспериментальных данных по формулам (1)-(5) определялось распределение полей остаточных пластических деформаций и напряжений. Далее на основании (6) задавались начальные псевдотемпературные деформации для конечно-элементной модели и численно решалась фиктивная задача термоупругости, в результате определялись остаточные (фиктивные температурные) напряжения.

На рис. 1, 2 в качестве примера приведены результаты расчёта для сплошных цилиндрических образцов из стали 45 и сплава Д16Т, упрочнённых гидродробеструйной обработкой. Из приведённых данных видно, что результаты расчётов, выполненных по по аналитическому решению (1)-(5) и МКЭ, близки между собой, и зачастую штрихпунктирная и сплошная линии в данном масштабе рисунков сливаются. Экспериментальные значения осевой компоненты $\sigma_{z}$ остаточных напряжений в упрочнённом слое, которые брались как исходная информация для построения аналитического решения, также близки к расчётным.

2. Решение задачи для образца с полукруглым надрезом, нанесённым после ОПД. Для решения задачи о перераспределении остаточных напряжений в образце с полукруглым надрезом радиуса $\rho$, нанесённым после ОПД (геометрическая схема представлена на рис. 3), предполагается, что в процессе перераспределения напряжений дополнительные пластические деформации не возникают, а финишные остаточные напряжения обусловлены упругим изменением объёма цилиндра. В этом случае возникает задача, аналогичная температурной в теории упругости.

Схема решения задачи для цилиндра с надрезом аналогична случаю гладкого образца и также состоит из трёх этапов:

1) разбиение цилиндрического образца с надрезом на конечные элементы;

2) задание начальных остаточных пластических деформаций псевдотемпературными деформациями в образце (на основании решения для гладкого образца);

3) решение задачи фиктивной термоупругости относительно неизвестных остаточных напряжений.

Первый и третий этапы решения задачи принципиально (технически) не отличаются от случая гладкого образца, а второй этап требует пояснения. На основании экспериментальной информации о распределении одной и/или двух компонент остаточных напряжений в упрочнённом слое гладкого образ- 


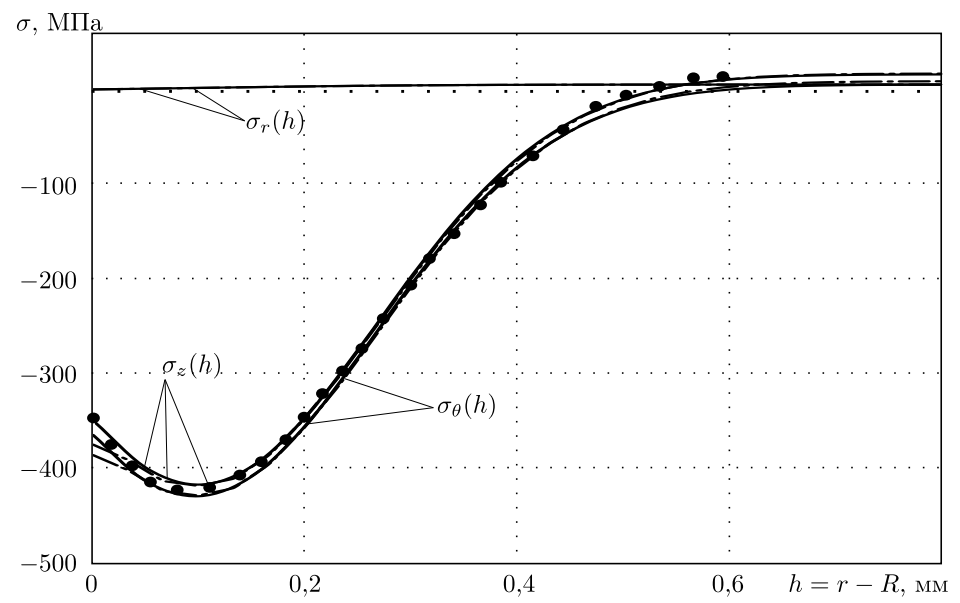

Рис. 1. Распределение остаточных напряжений по глубине $h$ упрочнённого слоя (сталь 45 , $R=25$ мм) : сплошная линия - расчёт по аналитическому решению (1)-(5); штрихпунктирная линия - расчёт МКЭ; точки - экспериментальные данные [4]

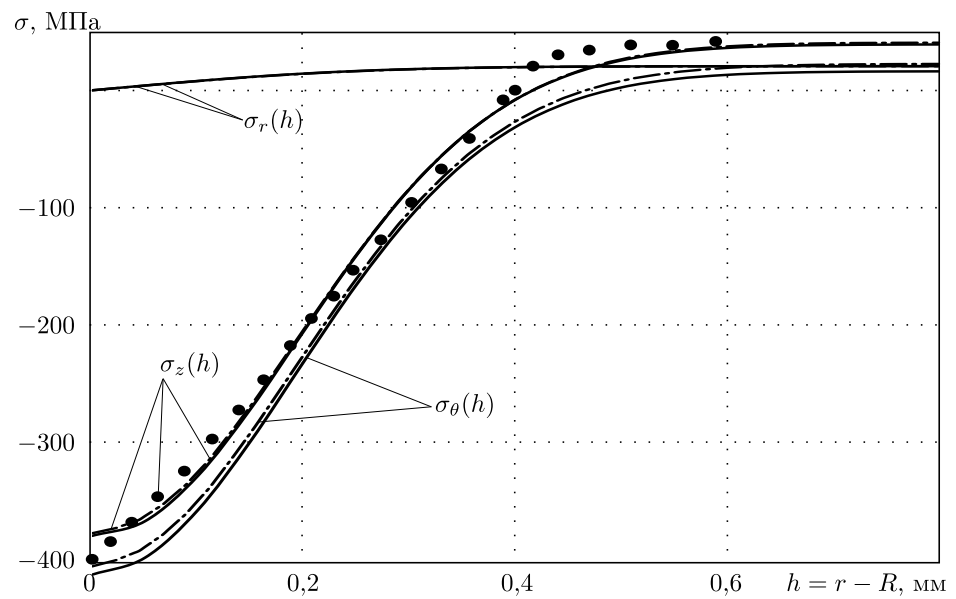

Рис. 2. Распределение остаточных напряжений по глубине $h$ упрочнённого слоя (сплав Д16Т, $R=5 \mathrm{Mм})$ : сплошная линия - расчёт по аналитическому решению (1)-(5); штрихпунктирная линия - расчёт МКЭ; точки - экспериментальные данные [4]

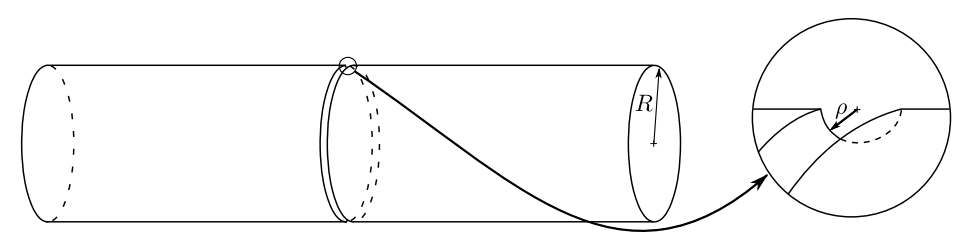

Рис. 3. Схема образца с круговым надрезом 
ца по формулам (2), (3) определяются начальные остаточные пластические деформаций $q_{i}(r)$, которые моделируются псевдотемпературными деформациями (6). Затем поле псевдотемпературных деформаций переносится на образец с надрезом.

Процесс решения данной задачи в вычислительном комплексе ANSYS также полностью автоматизирован.

На рис. 4 приведены результаты расчёта остаточных напряжений в цилиндрическом образце с круговым надрезом из стали 45 после процесса перераспределения остаточных напряжений от дна надреза по толщине слоя $h=R-\rho-r$ в наименьшем сечении детали, а на рис. 5 приведена аналогичная информация для образца с надрезом из сплава Д16Т.

Из сравнения данных следует, что для рассматриваемых образцов в кон-

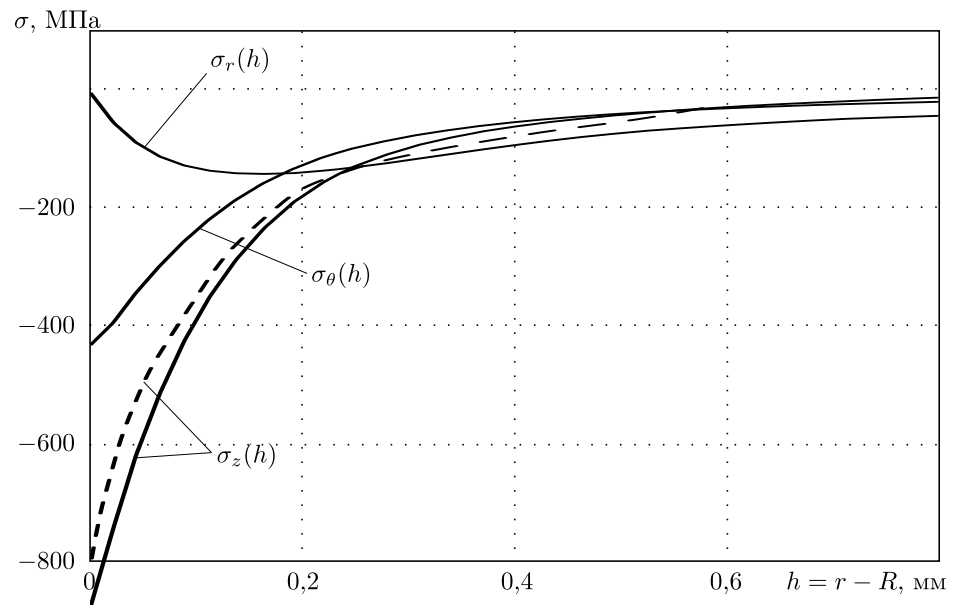

Рис. 4. Распределение остаточных напряжений от дна надреза по толщине слоя $h=R-$ $-\rho-r$ в наименьшем сечении детали (сталь $45, R=25$ мм, $\rho=0,3$ мм): сплошная линия расчёт МКЭ; штриховая линия-методика работы [4]

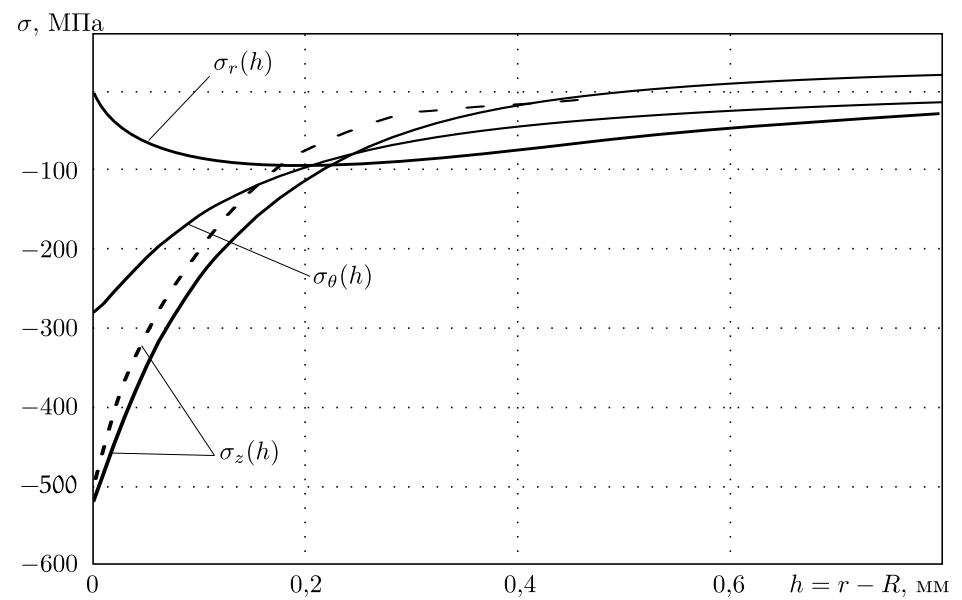

Рис. 5. Распределение остаточных напряжений от дна надреза по толщине слоя $h=R-$ $-\rho-r$ в наименьшем сечении детали (сплав Д16Т, $R=5 \mathrm{mм}, \rho=0,3 \mathrm{мм})$ : сплошная линия расчёт МКЭ; штриховая линия-методика работы [4] 
центраторе на дне надреза происходит существенное увеличение (по модулю) величины остаточного напряжения $\sigma_{z}$ по сравнению с этим же напряжением на поверхности гладкого упрочнённого образца. В то же время, напряжения $\sigma_{\theta}$ становятся несколько меньше (по модулю) на дне надреза по отношению к этому напряжению в гладком образце. Величина $\sigma_{r}$ численно (по модулю) существенно не изменяется, однако в концентраторе меняется знак этого напряжения. Для сопоставимости результатов выполнен сравнительный анализ расчётных значений для напряжения $\sigma_{z}$ по методике настоящей работы с данными расчёта этой компоненты по приближённой аналитической зависимости, полученной в $[4,7]$ методами теории функций комплексного переменного для плоской задачи, когда вместо всего цилиндрического образца рассматривалась тонкая пластина - осевое сечение цилиндра с концентратором напряжений. На рис. 4 и 5 данные расчёта величины $\sigma_{z}$ по методике $[4,7]$ приведены штриховой линией, при этом наблюдается соответствие данных расчёта по обеим методикам.

3. Заключение. В отличие от известных методов решения поставленной в работе задачи разработанный метод позволяет учитывать реальное неоднородное по радиусу распределение остаточных пластических (начальных) деформаций. Кроме этого предложенный метод позволяет восстановить полную трёхмерную картину напряжённо-деформированного состояния в упрочнённых цилиндрических изделиях (гладких и с концентраторами в виде надрезов). Разработан программный продукт, позволяющий автоматически генерировать код для его последующего выполнения в ANSYS.

Работа выполнена при поддержке Министерства образования и науки РФ в рамках АВЦП «Развитие научного потенциала высшей школы» (проект 2.1.1/13944).

\section{БИБЛИОГРАФИЧЕСКИЙ СПИСОК}

1. Кравченко Б.А., Круиило В.Г., Гутман Г.Н. Термопластическое упрочнение-резерв повышения прочности и надёжности машин. Самара: СамГТУ, 2000. 215 с. [Kravchenko B.A., Krutsilo V.G., Gutman G.N. Thermoplastic hardening is reserve for increasing the strength and reliability of the machines. Samara: SamGTU, 2000. 215 pp.]

2. Мифтахов А. А., Мазеин П. Г. Моделирование остаточных напряжений при гидродробеструйной обработке// Извест. Челябин. научн. иентра УрО РАН, 2006. № 4(34). C. 38-42. [Miftakhov A. A., Mazein P. G. Modeling of residual stresses in hydro-shot-blasting processing // Izvest. Chelyabin. Nauchn. Centra UrO RAN, 2006. no. 4(34). Pp. 38-42].

3. Вакулюк В.С., Сазанов В.П., Филиппов А.А., Мамедов А.И. Моделирование упрочнения малых по протяжённости зон цилиндрических изделий/ В сб.: Труды седъмой Всероссийской научной конференции с международным участием. Часть 1: Математические модели механики, прочности и надёжности элементов конструкций/ Матем. моделирование и краев. задачи. Самара: СамГТУ, 2010. С. 87-90. [Vakulyuk V.S. Sazanov V.P. Filippov A. A. Mamedov A. I. Modeling of small areas by the length hardening for cylindrical products/ In: Proceedings of the Seventh All-Russian Conference with international participation. Part 1/ Matem. Mod. Kraev. Zadachi. Samara: SamGTU, 2010. Pp. 87-90].

4. Павлов В.Ф., Столяров А.К., Вакулюк В.С., Кирпичёв В.А. Расчёт остаточных напряжений в деталях с концентраторами напряжений по первоначальным деформациям. Самара: СНЦ РАН, 2008. 124 c. [Pavlov V. F. Stolyarov A. K. Vakulyuk V. S. Kirpichev V. A. Residual stresses calculation in products with stress concentrators on the initial deformation. Samara: SNC RAN, 2008. 124 pp.]

5. Блюменштейн В. Ю., Махалов М. С. Расчётная модель остаточных напряжений упрочненного поверхностного слоя при размерном совмещенном обкатывании // Вестн. Куз- 
бас. гос. техн. ун-та, 2008. №5. C. 50-58. [Blyumenshtejn V. Yu., Makhalov M.S. Computational model of residual stresses of the hardened surface layer at dimensional joint runningin // Vestn. Kuzbas. Gos. Tekhn. Un-ta, 2008. no. 5. Pp. 50-58].

6. Саушкин М.Н., Радченко В. П., Павлов В.Ф. Метод расчёта полей остаточных напряжений и пластических деформаций в цилиндрических образцах с учётом анизотропии процесса поверхностного упрочнения // ПМТФ, 2011. Т. 52, № 2. С. 173-182; англ. пер.: Saushkin M.N., Radchenko V.P., Pavlov V.F. Method of calculating the fields of residual stresses and plastic strains in cylindrical specimens with allowance for surface hardening anisotropy // J. Appl. Mech. Tech. Phys., 2011. Vol. 52, no. 2. Pp. 303-310.

7. Иванов С.И., Шатунов М. П., Павлов В.Ф. Влияние остаточных напряжений на выносливость образцов с надрезом / В сб.: Вопросъ прочности элементов авиационных конструкиий. Вып. 1. Куйбышев: КуАИ, 1974. С. 88-95. [Ivanov S. I., Shatunov M. P., Pavlov V.F. Effect of residual stresses on the fatigue of noth specimens / In: Problems of aircraft structure element strength. Issue 1. Kuibyshev: KuAI, 1974. Pp. 88-95].

Поступила в редакцию $13 / \mathrm{V} / 2011$;

в окончательном варианте - 13/VI/2011.

MSC: 74G70;74S05

FINITE ELEMENT MODELING OF RESIDUAL STRESS DISTRIBUTION IN SOLID HARDENED CYLINDRICAL SAMPLES AND SAMPLES WITH SEMICIRCULAR NOTCH

\section{N. Saushkin, A. Yu. Kurov}

Samara State Technical University,

244, Molodogvardeyskaya st., Samara, 443100, Russia.

E-mails: msaushkin@gmail.com, alexeykurov@gmail.com

We give a method for solving the problem of the distribution of residual stresses in a continuous surface hardened cylindrical samples and samples with a semicircular notch based on the finite element method. As the initial information the experimentally determined alone and / or two components of residual stresses in a hardened layer is used. By the example of a cylindrical specimen made of St. 45 and D16T steels the results of calculations performed by the proposed method are compared with the experimental data and the results of calculations by other methods.

Key words: residual stresses distribution, cylindrical specimen, specimen with semicircular notch, finite element method.

Original article submitted 13/V/2011; revision submitted $13 / \mathrm{VI} / 2011$.

Mikhail N. Saushkin (Ph. D. (Phys. \& Math.)), Doctoral Candidate, Dept. of Applied Mathematics \& Computer Science. Alexey Yu. Kurov, Student, Dept. of Applied Mathematics \& Computer Science. 